\title{
Bunching coefficients in echo-enabled harmonic generation
}

\author{
G. Dattoli* and E. Sabia ${ }^{\dagger}$ \\ ENEA-Centro Ricerche Frascati, via E. Fermi, 45, IT 00044 Frascati, Rome, Italy
}

(Received 21 May 2013; published 24 July 2013)

\begin{abstract}
Coulombian diffusion determines a dilution of bunching coefficients in free electron laser seeded devices. From the mathematical point of view the effect can be modeled through a heat-type equation, which can be merged with the ordinary Liouville equation, ruling the evolution of the longitudinal phase space beam distribution. We will show that the use of analytical tools like the generalized Bessel functions and algebraic techniques for the solution of evolution problems may provide a useful method of analysis and shine further light on the physical aspects of the underlying mechanisms.
\end{abstract}

DOI: 10.1103/PhysRevSTAB.16.070702

\section{INTRODUCTION}

In this paper we will pursue some technical details concerning the computation and the physical understanding of the recent analysis by Stupakov [1] on the effect of Coulomb diffusion on bunching coefficients in echoenabled harmonic generation (EEHG) free electron laser (FEL) seeded devices [2]. The considerations developed in this paper should be understood as a complement to Refs. [1,2], with the aim of providing a more general computational framework, benefiting from the formalism of beam transport employing algebraic means [3]. In this treatment the transport through magnetic elements is treated in terms of an exponential operator acting on an initial phase space distribution and usually does not contain "transport" elements provided by a heat-type diffusion. Here we will show that diffusion mechanisms can be included in such a framework in a fairly straightforward way [4], preserving the spirit of exponential operator concatenation.

The effect of the Coulomb diffusion on the e-beam longitudinal phase space distribution is ruled, according to $[1,2]$, by the equation (where $D$ has the dimension of the inverse of a length and $s$ the dimension of a length)

$\partial_{s} f(p, \zeta, s)=D \partial_{p}^{2} f(p, \zeta, s), \quad f(p, \zeta, 0)=f_{0}(p, \zeta)$,

where $s$ is the coordinate of propagation, $\zeta$ is the longitudinal coordinate in the beam, $p$ is associated to the beam energy, $D$ is the Coulomb diffusion coefficient, and $f_{0}(p, \zeta)$ is the "initial" distribution. We will discuss the physical meaning of the previous quantities and we will provide their explicit expression in the following; for the moment we note that, having assumed that $D$ is

\footnotetext{
*dattoli@frascati.enea.it
}

†sabia@frascati.enea.it

Published by the American Physical Society under the terms of the Creative Commons Attribution 3.0 License. Further distribution of this work must maintain attribution to the author(s) and the published article's title, journal citation, and DOI.
PACS numbers: 41.60.Cr, 41.85.Ja, 02.30.Uu, 02.30.Vv

independent of $p$, we can solve (1.1) via the following Gauss-Weierstrass (G-W) transform [5]:

$f(p, \zeta, s)=\frac{1}{2 \sqrt{\pi D s}} \int_{-\infty}^{\infty} e^{-\left[(p-\eta)^{2} /(4 D s)\right]} f_{0}(\eta, \zeta) d \eta$.

The bunching coefficients can be defined as those of the Fourier components of the expansion [6]

$$
f(p, \zeta, s)=\sum_{n=-\infty}^{+\infty} b_{n}(p, s) e^{i n \zeta}
$$

and are, therefore, specified by

$$
b_{m}(p, s)=\frac{1}{2 \pi} \int_{0}^{2 \pi} d \zeta f(p, \zeta, s) e^{-i m \zeta} .
$$

The dilution of the bunching coefficients induced by the Coulomb diffusion can therefore be mathematically expressed as

$$
b_{m}(p, s)=\frac{1}{2 \sqrt{\pi D s}} \int_{-\infty}^{\infty} e^{-\left[(p-\eta)^{2} /(4 D s)\right]} b_{m}(\eta) d \eta .
$$

Following Ref. [1], we assume the initial distribution

$$
f_{0}(p, \zeta)=\frac{1}{\sqrt{2 \pi}} e^{-\left\{\left[p-A_{1} \sin \left(\zeta-B_{1} p\right)\right]^{2} / 2\right\}}
$$

whose physical meaning will be discussed in the forthcoming section. The solution of Eq. (1.1) can be therefore easily computed, even though it cannot be obtained in analytical form. In Fig. 1 we have reported the effect of the dispersion on the initial distribution [Fig. 1(a)], after a drift $s=30$ with $D=7 \times 10^{-3}$ [Fig. 1(b)].

It is evident that the Coulomb term smears out the oscillations, associated with the bunching coefficients, which tend to disappear or to be strongly suppressed, as we will further discuss in the following. If we neglect in Eq. (1.6) the contributions $\left[A_{1} \sin \left(\zeta-B_{1} p\right)\right]^{2}$ we can expand $f_{0}(p, \zeta)$ in series of Bessel functions, namely, 

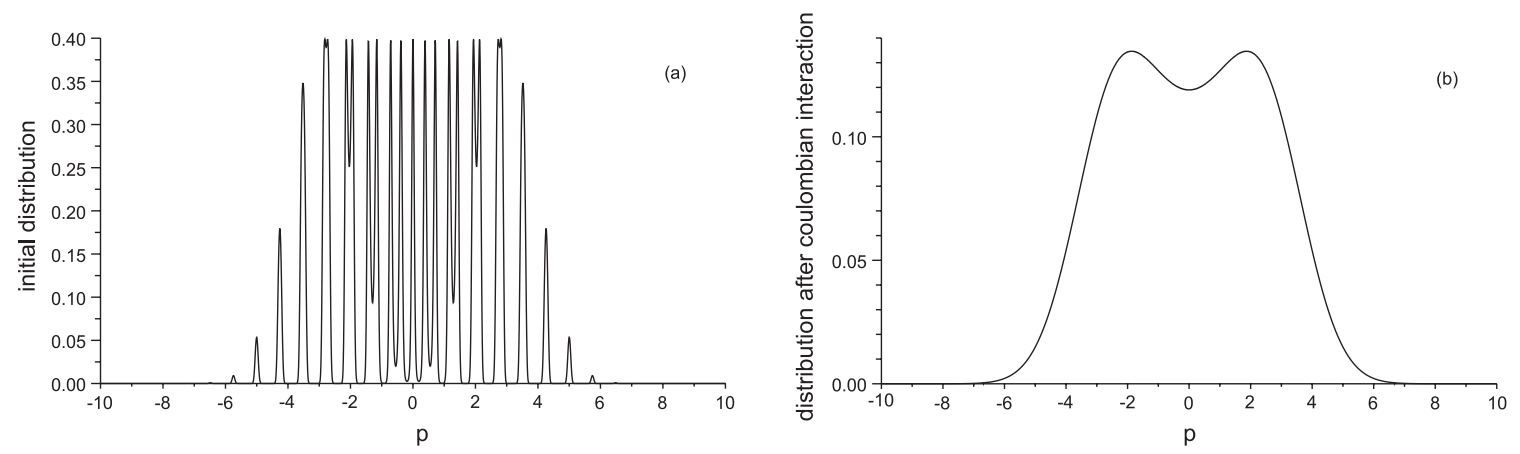

FIG. 1. Evolution of the distribution function $f(p, \zeta, s)$ undergoing the Coulombian diffusion. (a) $f(p, 0,0), A_{1}=3, B_{1}=8.47$, and (b) $f(p, 0,30), A_{1}=3, B_{1}=8.47, D=7 \times 10^{-3}$.

$$
\begin{aligned}
f_{0}(p, \zeta) & \cong \frac{1}{\sqrt{2 \pi}} e^{-\left(p^{2} / 2\right)} e^{A_{1} p \sin \left(\zeta-B_{1} p\right)} \\
& =\frac{1}{\sqrt{2 \pi}} e^{-\left(p^{2} / 2\right)} \sum_{n=-\infty}^{\infty} e^{i n\left(\zeta-B_{1} p\right)} J_{n}\left(-i A_{1} p\right),
\end{aligned}
$$

where $J_{n}(\cdot)$ are cylindrical Bessel functions of the first kind. The associated bunching coefficients are, neglecting contributions in $A_{1}^{2}$, provided by

$$
\begin{aligned}
b_{m}(p) & =\frac{1}{2 \pi} \int_{0}^{2 \pi} f_{0}(p, \zeta) e^{-i m \zeta} d \zeta \\
& =\frac{1}{\sqrt{2 \pi}} e^{-\left(p^{2} / 2\right)} e^{-i m B_{1} p} J_{m}\left(-i A_{1} p\right) .
\end{aligned}
$$

The G-W transform can be accordingly written as (see the Appendix)

$$
\begin{aligned}
f(p, \zeta, s) \cong & \frac{1}{2 \sqrt{2 \pi} \sqrt{1+2 D s}} e^{-\left\{p^{2} /[2(1+2 D s)]\right\}} \\
& \times \sum_{n=-\infty}^{\infty} e^{-\left\{\left[\left(n B_{1}\right)^{2} D s\right] /(1+2 D s)\right\}} e^{\left(i n B_{1} p\right) /[2(1+2 D s)]} \\
& \times e^{i n \zeta} J_{n}\left(\frac{A_{1}\left(n B_{1} D s-i p\right)}{1+2 D s}\right)
\end{aligned}
$$

and bunching coefficients now read

$$
\begin{aligned}
b_{m}(p, s) \cong e^{-\left\{\left[\left(m B_{1}\right)^{2} D s\right] /(1+2 D s)\right\}} \Phi_{m}, \\
\Phi_{m}=\frac{1}{2 \sqrt{2 \pi} \sqrt{1+2 D s}} e^{-\left\{p^{2} /[2(1+2 D s)]\right\}} \\
\quad \times e^{\left(i m B_{1} p\right) /[2(1+2 D s)]} J_{m}\left(\frac{A_{1}\left(m B_{1} D s-i p\right)}{1+2 D s}\right) .
\end{aligned}
$$

The effect of the Coulombian diffusion is therefore twofold: it induces (a) a dispersion due to the term $2 D s$, and (b) a suppression of the higher orders harmonics occurring through $e^{-\left\{\left[\left(m B_{1}\right)^{2} D\right] s /(1+2 D s)\right\}}$.

The suppression factor can be written as $e^{-\left(m^{2} / L\right)\left\{s /\left[1+\left(2 / B_{1}^{2} L\right) s\right]\right\}}$, where $L=1 / B_{1}^{2} D ;$ it coincides with the analogous expression given in Ref. [2], provided that $\frac{2}{B_{1}^{2} L} s \ll 1$.
The use of a one-dimensional model, employing the simple diffusion equation (1.1), to study the effect of Coulomb diffusion on bunching, may sound simplistic. A more realistic analysis should demand for the inclusion of the interplay between transverse and longitudinal phase space dynamics, which has been summarized by the coefficient $D$, assumed to be independent of the longitudinal coordinate $s$ and of the energy variable. We have followed the approximations of Refs. [2,7], according to which an average is taken on the transverse phase space. Such a procedure is justified by the operating conditions of an electron beam driving $x$-ray FEL operation, in which the equivalent longitudinal temperature is much less than its transverse counterpart. It must also be stressed that the bunching coefficients are calculated here using a 1D treatment and therefore, at a certain level of the analysis, an averaging procedure is mandatory. The situation is not dissimilar from the approximation used for the evaluation of the inhomogeneous broadening effects, due to the transverse e-beam emittances. In 1D FEL theory they are transformed into longitudinal effects, by a proper redefinition of the relative energy spread, ensured by an appropriate averaging on the transverse phase space. The method we are developing can accordingly be viewed as an evaluation of the inhomogeneous broadening induced on the bunching coefficients by the energy spread due to the Coulombian diffusion.

In these introductory remarks we have provided a few remarks on the type of formalism we are going to use to treat the bunching mechanism in EEHG FEL seeded devices; the forthcoming sections will cover more physical details.

\section{LIOUVILLE AND VLASOV OPERATORS AND BUNCHING COEFFICIENT DYNAMICS}

We have quoted the initial distribution given in Eq. (1.6) without any comment about its physical meaning. Its origin can be traced back to the following Liouville equation:

$$
\begin{gathered}
\partial_{s} f(p, \zeta, s)=\hat{L} f(p, \zeta, s), \quad \hat{L}=-B p \partial_{\zeta}+A V^{\prime}(\zeta) \partial_{p}, \\
f_{0}(p, \zeta)=\frac{1}{\sqrt{2 \pi}} e^{-\left(p^{2} / 2\right)},
\end{gathered}
$$


which rules the evolution of an ensemble of noninteracting particles, driven by the Hamiltonian

$$
H=B \frac{p^{2}}{2}+A V(\zeta)
$$

The solution of Eq. (2.1) can be cast in the form

$$
f(p, \zeta, s)=\hat{U}(s) f_{0}(p, \zeta), \quad \hat{U}(s)=e^{\hat{L} s} .
$$

We have denoted by $\hat{U}(s)$ and $\hat{L}$ the evolution and Liouville operators, respectively, of our dynamical problem $[3,4]$. We note that the Liouville operator breaks into two noncommuting parts, therefore any treatment of the associated evolution operator demands for an approximate disentanglement of the exponential, which, for simplicity will be assumed to be provided by $[3,4]$

$$
e^{\left[-B p \partial_{\zeta}+A V^{\prime}(\zeta) \partial_{p}\right] s} \cong e^{-B s p \partial_{\zeta}} e^{A s V^{\prime}(\zeta) \partial_{p}}
$$

which is accurate to $\frac{A B}{2} s^{2}\left[p \partial_{\zeta}, V^{\prime}(\zeta) \partial_{p}\right]$.

The use of the rule $e^{\lambda \partial_{x}} f(x)=f(x+\lambda)$ allows one to cast the solution of Eq. (2.1) in the form

$$
\begin{aligned}
f(p, \zeta, s) & \cong e^{-B s p \partial_{\zeta}} e^{A s V^{\prime}(\zeta) \partial_{p}} f_{0}(p, \zeta) \\
& =e^{-B s p \partial_{\zeta}} f_{0}\left[p+A s V^{\prime}(\zeta), \zeta\right] \\
& =f_{0}\left[p+A s V^{\prime}(\zeta-B s p), \zeta-B s p\right] .
\end{aligned}
$$

Therefore by setting

$$
V(\zeta)=\cos (\zeta), \quad A s=A_{1}, \quad B s=B_{1}
$$

and keeping as initial distribution a Gaussian in $p$, we recover Eq. (1.6).

The physical scheme we are dealing with is reported in Ref. [2], to which the reader is addressed for further details. The e-beam undergoes two successive modulations induced by two different lasers in two different sections. The beam initial distribution (1.6) is that occurring after the first chicane at the entrance of the second modulator.

The physical content of the previous variables is the following:

$$
A_{1}=\frac{\Delta E_{1}}{\sigma_{E}}, \quad B_{1}=R_{5,6} \frac{k_{L} \sigma_{E}}{E_{0}}, \quad p=\frac{E-E_{0}}{\sigma_{E}}, \quad \zeta=k_{L} z
$$

where $\sigma_{E}$ is the e-beam energy spread, $\Delta E_{1}$ is the induced energy modulation, and $k_{L}$ is the laser wave vector.

The dynamics of the bunching coefficients can be obtained from Eqs. (2.1) and (1.3) as $\left[b_{n}(p, s)=b_{n}\right]$

$$
\begin{aligned}
\partial_{s} b_{n} & =-i \text { Bpnb }_{n}+\frac{1}{2 i} A\left[b_{n-1}-b_{n+1}\right], \\
b_{n}(p, 0) & =\delta_{n, 0} e^{-\left(p^{2} / 2\right)}
\end{aligned}
$$

Equation (2.8) yields an idea of the coupling between the various bunching coefficients and has already been derived in a closely similar form in Ref. [6].
The inclusion of the Coulomb diffusion in this scheme can be modeled by transforming the Liouville into a Vlasov equation, namely,

$$
\partial_{s} f(p, \zeta, s)=\left(D \partial_{p}^{2}+\hat{L}\right) f(p, \zeta, s) .
$$

The diffusion coefficient, expressed in practical units, reads [2]

$$
D=1.55 \frac{I[\mathrm{kA}]}{\varepsilon_{x}[\mu \mathrm{m}] \sigma_{x}[100 \mu \mathrm{m}]\left(\sigma_{E}[\mathrm{keV}]\right)^{2}}
$$

with $\varepsilon_{x}$ and $\sigma_{x}$ being the emittance and beam section, respectively

The solution of Eq. (2.9) can be obtained as

$$
f(p, \zeta, s) \cong e^{D s \partial_{p}^{2}}\left[e^{s \hat{L}} f_{0}(p, \zeta)\right]
$$

According to this approximation the effect of the diffusion is calculated separately from that induced by the Liouvillian contribution. Higher order disentanglements $[3,4]$ can be used to get more accurate results, but the present approximation is adequate for our purposes.

In Fig. 2 we report the contour plots of the Liouville distribution with and without the effect of the Coulombian diffusion for the same $s$ value. It is evident that the presence of a nonzero $D$ value provides a significant reduction of the distribution harmonic content. A global view is provided by Fig. 3 where we have reported the 3D plot of the Liouville distribution under the action of diffusion for different $s$ values.

We have so far assumed that the coefficients $A$ and $B$ in the Hamiltonian (2.2) are independent of the longitudinal coordinate, but this is not strictly true. If we include such a dependence, the evolution operator in Eq. (2.3) is no more sufficient to provide the correct description of the solution of the Liouville problem. The Liouvillian is indeed time $(s)$ dependent and the associated evolution operator should be computed by including time ordering contributions [8]. The error induced by neglection of the time ordering corrections is noninfluential, since it is smaller than the intrinsic error of the ordering procedure, adopted in the disentanglement (2.4), associated with the noncommutativity between kinetic and potential parts of the Liouvillian. If higher order disentanglement schemes with smaller intrinsic error are used, the time ordering corrections must be included. If we consider the effects of bunching and dispersion occurring at different points in the transfer line, as it happens in practice, the disentanglement procedure is not strictly necessary and therefore the ordering (2.4) can be considered exact, provided that we neglect the kinetic effects inside the undulator.

The nice feature of the procedure we are adopting is its modularity, which will be further appreciated in the forthcoming section. 
(a)

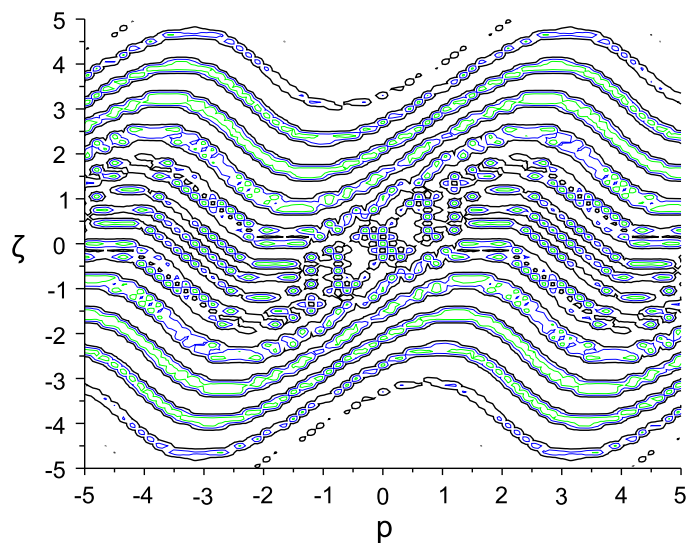

(b)

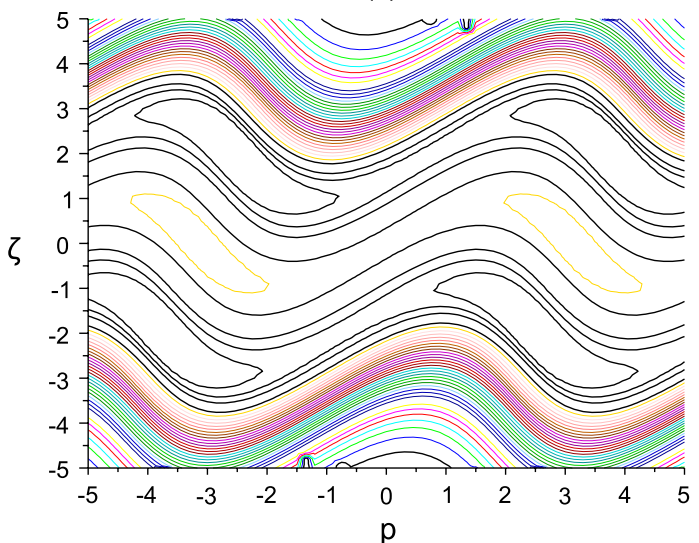

FIG. 2. Liouville distribution contour plots: (a) without Coulombian diffusion, (b) with Coulombian diffusion $D=7 \times 10^{-3}$.
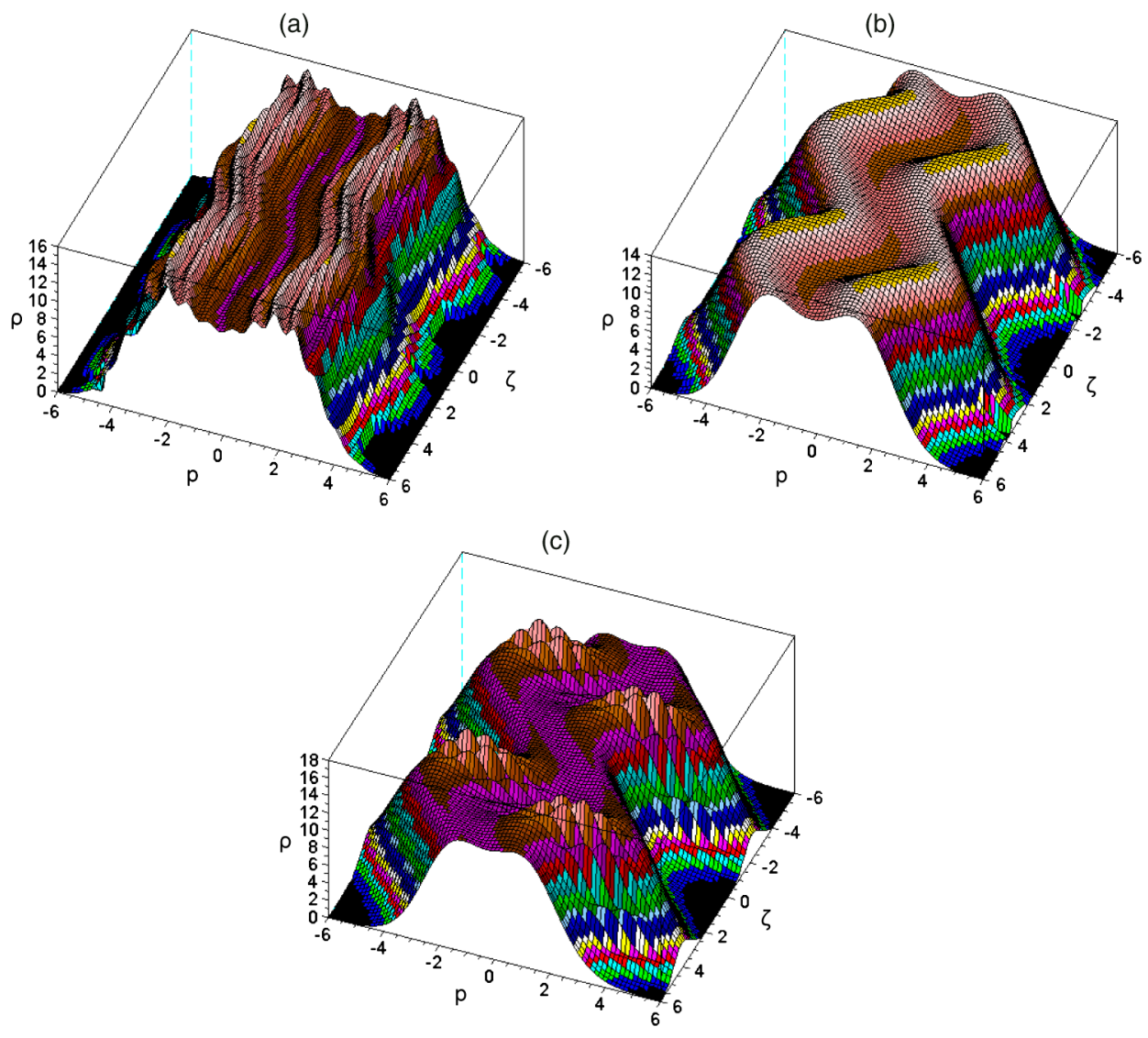

FIG. 3. Liouville distribution under the action of the Coulombian diffusion $\left(D=7 \times 10^{-3}\right)$ for different $s$ values: (a) $s=6$, (b) $s=20$, (c) $s=30$.

\section{GENERALIZED BESSEL FUNCTIONS AND BUNCHING COEFFICIENT DYNAMICS}

In the Introduction, we have expanded the initial distribution, by neglecting the contributions in $A^{2}$. Such an approximation implies that the induced energy modulation is not large compared to the natural e-beam energy spread. However, if we relax these assumptions and employ the formalism of generalized Bessel functions [5], we can obtain a more general view on the problem under study. It has indeed been shown that two variable Bessel functions can be expressed through the generating function, 


$$
\begin{aligned}
e^{i x \sin (\vartheta)-y[\sin (\vartheta)]^{2}} & =\sum_{n=-\infty}^{+\infty} e^{i n \vartheta} J_{n}(x, y), \\
J_{n}(x, y) & =\sum_{r=0}^{\infty} \frac{(-1)^{r}}{2^{n+2 r}} \frac{H_{n+2 r}(x, y)}{r !(n+r) !}, \\
H_{n}(x, y) & =n ! \sum_{r=0}^{n / 2} \frac{x^{n-2 r} y^{r}}{r !(n-2 r) !},
\end{aligned}
$$

where $H_{n}(x, y)$ are two variable Hermite-Kampè dè Fèrièt polynomials [5]. They are actually understood as belonging to the family of Hermite based functions and are widely exploited to deal with radiation scattering problems [9], going beyond the dipole approximation.

It is also worth noting the following operational property [5]: ${ }^{1}$

$$
{ }_{H} J_{n}(x, y)=e^{y \partial_{x}^{2}} J_{n}(x)
$$

which will be used in the following.

The inclusion of the generalized Bessel functions does not change substantively the form of the bunching coefficients, which are obtained from Eqs. (1.9) and (1.10) by replacing the Bessel function with

$$
{ }_{H} J_{n}\left(\frac{A_{1}\left(n B_{1} D s-i p\right)}{1+2 D s}, \frac{A_{1}^{2}}{1+2 D s}\right) \text {. }
$$

In the second modulator the initial distribution will be provided by the properly modified Eq. (1.9) so that

$$
f_{2}(p, \zeta) \cong e^{-B_{2} p \partial_{\zeta}} e^{-A_{2} \sin (\zeta) \partial_{p}} f_{1}(p, \zeta)
$$

which holds if the laser, in the second modulator, is the same as the first. We have removed the $s$ variable because it absorbed into the $A$ and $B$ terms. If we use the Bessel function expansion

$$
e^{-A_{2} \sin (\zeta) \partial_{p}}=\sum_{n=-\infty}^{\infty} e^{i n \zeta} J_{n}\left(i A_{2} \partial_{p}\right)
$$

we can therefore cast the distribution at the end of the second modulator as

$$
\begin{aligned}
f_{2}(p, \zeta) \cong & \sum_{n=-\infty}^{\infty} e^{i n\left(\zeta-B_{2} p\right)} J_{n}\left(i A_{2} \partial_{p}\right) f_{1}\left(p, \zeta-B_{2} p\right) \\
= & \frac{1}{\sqrt{2 \pi}} \sum_{n=-\infty}^{\infty} e^{i n\left(\zeta-B_{2} p\right)} J_{n}\left(i A_{2} \partial_{p}\right) \\
& \times e^{-\left(p^{2} / 2\right)} \sum_{m=-\infty}^{\infty} e^{i m\left[\zeta-\left(B_{1}-B_{2}\right) p\right]} J_{m}\left(-i A_{1} p\right) .
\end{aligned}
$$

\footnotetext{
${ }^{1}$ According to the property (3.2) the functions ${ }_{H} J_{n}(x, y)$ are solutions of the heat equation $\partial_{y} F(x, y)=\partial_{x}^{2} F(x, y), F(x, 0)=$ $J_{n}(x)$.
}

By rearranging the indices we end up with

$$
\begin{aligned}
f_{2}(p, \zeta) \cong & \frac{1}{\sqrt{2 \pi}} \sum_{l=-\infty}^{\infty} e^{i l \zeta} Q_{l} \\
Q_{l}= & \sum_{r=0}^{l} e^{-i(l-r) B_{2} p} J_{l-r}\left(i A_{2} \partial_{p}\right) e^{-\left(p^{2} / 2\right)} \\
& \times e^{-i(l-r)\left(B_{1}+B_{2}\right) p} J_{r}\left(-i A_{1} p\right) .
\end{aligned}
$$

According to such a picture the $n$th bunching coefficient is characterized by a discrete convolution of the bunching terms of the second modulator on the first.

The effect of the Coulombian diffusion on the distribution (3.6) can be easily computed, either numerically or analytically. By keeping the lowest order contribution $l=1$ and by approximating the Bessel function as

$$
J_{0}(x) \cong 1, \quad J_{1}(x) \cong \frac{x}{2},
$$

we obtain

$$
\begin{aligned}
Q_{1} \cong & -\left[\left(\frac{i A_{1} p}{2}\right)+\left(i A_{2} p\right) e^{-i\left(B_{1}+2 B_{2}\right) p}\right. \\
& \left.-\frac{B_{1}+B_{2}}{2} A_{2} e^{-i\left(B_{1}+2 B_{2}\right) p}\right] e^{-\left(p^{2} / 2\right)}
\end{aligned}
$$

From the above equation we easily deduce that the Coulombian contribution yields a suppression factor proportional to $e^{\left.-\left\{\left(B_{1}+2 B_{2}\right)^{2} D s\right] /(1+4 D s)\right\}}$. More in general as also pointed out in Ref. [1], the larger is the order of bunching the more significant is the effect of the reduction.

The bunching coefficients vs $s$ in the presence of diffusion is provided by Fig. 4 .

The formalism we have presented in this paper provides a fairly detailed analysis of how the Coulombian diffusion affects the bunching coefficients in echo-enabled FEL devices. Our treatment is complementary to the seminal contributions of Refs. [1,2] and confirm, within a more general framework, their conclusions. We believe that the

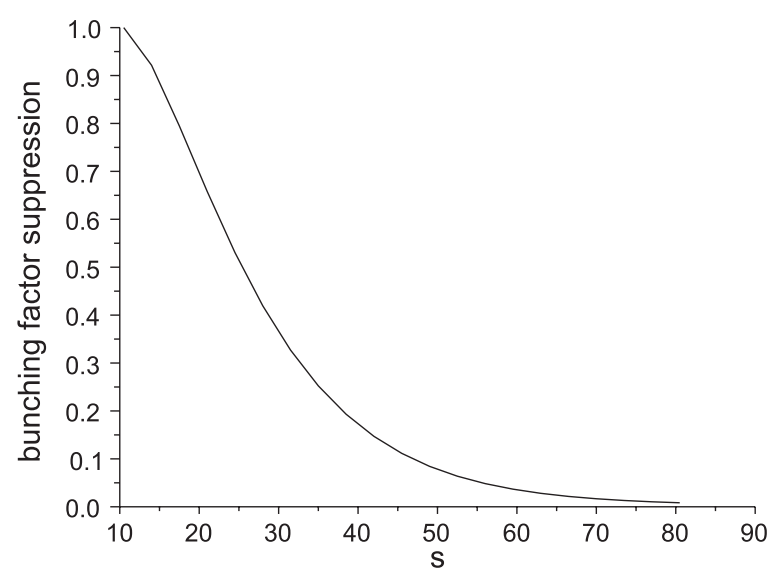

FIG. 4. Effect of the Coulombian diffusion $\left(D=3.5 \times 10^{-4}\right)$ on the bunching coefficient $(m=9) b_{9}(D) / b_{9}(0)$ vs $s$. 
modularity of the methods we have described in this paper offer further opportunities as e.g. that of including in the treatment more complicated arrangements of transport lines and of external fields. The possibility of embedding these procedures with diffusion and damping in storage rings will be discussed elsewhere.

\section{APPENDIX}

We will discuss here some technical details concerning the solution of the heat equations

$$
\partial_{t} F(x, t)=\partial_{x}^{2} F(x, t), \quad F(x, 0)=x^{n} e^{-x^{2}}
$$

which can formally be written as

$$
F(x, t)=e^{t \partial_{x}^{2}}\left(x^{n} e^{-x^{2}}\right) .
$$

The use of operational methods [5] yields

$$
F(x, t)=\left(e^{t \partial_{x}^{2}} x^{n} e^{-t \partial_{x}^{2}}\right) e^{t \partial_{x}^{2}} e^{-x^{2}}=\left(x+2 t \partial_{x}\right)^{n} e^{t \partial_{x}^{2}} e^{-x^{2}} .
$$

Then by applying the Glaisher and Burchnall [5] rules, respectively reported below,

$$
\begin{aligned}
e^{t \partial_{x}^{2}} e^{-x^{2}} & =\frac{1}{\sqrt{1+4 t}} e^{-\left[x^{2} /(1+4 t)\right]}, \\
\left(x+2 t \partial_{x}\right)^{n} & =\sum_{s=0}^{n}\left(\begin{array}{l}
n \\
s
\end{array}\right) H_{n-s}(x, t)\left(2 t \partial_{x}\right)^{s}
\end{aligned}
$$

provides the solution of our problem in the form

$$
F(x, t)=\frac{1}{\sqrt{1+4 t}} \sum_{s=0}^{n}\left(\begin{array}{c}
n \\
s
\end{array}\right) H_{n-s}(x, t)\left(2 t \partial_{x}\right)^{s} e^{-\left[x^{2} /(1+4 t)\right]} .
$$

The identities [5]

$$
\begin{aligned}
\partial_{x}^{s} e^{-\alpha x^{2}} & =(-1)^{s} H_{s}(-2 \alpha x,-\alpha), \\
H_{n}\left(\alpha_{1}+\alpha_{2}, \beta_{1}+\beta_{2}\right) & =\sum_{s=0}^{n}\left(\begin{array}{l}
n \\
s
\end{array}\right) H_{n-s}\left(\alpha_{1}, \beta_{1}\right) H_{s}\left(\alpha_{2}, \beta_{2}\right)
\end{aligned}
$$

finally provide the result

$$
\begin{aligned}
F(x, t)= & \frac{1}{\sqrt{1+4 t}} \sum_{s=0}^{n}\left(\begin{array}{l}
n \\
s
\end{array}\right) H_{n-s}(x, t) H_{s}\left(-\frac{4 x t}{1+4 t},-\frac{4 t^{2}}{1+4 t}\right) \\
& \times e^{-\left[x^{2} /(1+4 t)\right]} \\
= & \frac{1}{\sqrt{1+4 t}} H_{n}\left(\frac{x}{1+4 t}, \frac{t}{1+4 t}\right) e^{-\left[x^{2} /(1+4 t)\right]} .
\end{aligned}
$$

It is accordingly evident that for any initial condition such that

$$
F(x, 0)=g(x) e^{-x^{2}}
$$

with

$$
\begin{aligned}
g(x) & =\sum_{p} a_{p} x^{p}, \\
F(x, t) & =\frac{1}{\sqrt{1+4 t}} \sum_{p} a_{p} H_{p}\left(\frac{x}{1+4 t}, \frac{t}{1+4 t}\right) e^{-\left[x^{2} /(1+4 t)\right]} .
\end{aligned}
$$

[1] G. Stupakov, Phys. Rev. Lett. 102, 074801 (2009).

[2] G. Stupakov, in Proceedings of the FEL2011 Conference, Shanghai, China, 2011 [http://www.jacow.org].

[3] D. Babusci, G. Dattoli, M. Quattromini, and E. Sabia, Phys. Rev. E 87, 033202 (2013), and references therein.

[4] A. J. Dragt, Lie Methods for Nonlinear Dynamics with Application to Accelerator Physics (University of Maryland, College Park, MD, 2013).

[5] G. Dattoli, P. L. Ottaviani, A. Torre, and L. Vazquez, Riv. Nuovo Cimento Soc. Ital. Fis. 20, 1 (1997).

[6] G. Dattoli, L. Giannessi, and A. Torre, J. Opt. Soc. Am. B 10, 2136 (1993).

[7] V. Lebedev, AIP Conf. Proc. 773, 440 (2005).

[8] G. Dattoli, M. Quattromini, A. Torre, and L. Vazquez, Phys. Lett. A 240, 213 (1998); S. Blanes, F. Casas, J. A. Oteo, and J. Ros, Phys. Rep. 470, 151 (2009).

[9] H. R. Reiss, Phys. Rev. A 22, 1786 (1980). 